\title{
Designing Smart Energy Network Ecosystem for Integrated energy services in urban areas*
}

\author{
Chunyan Dai, Min Tang, Ying Liu, Jijiang He, Zhongqing Yang and Ying Yang
}

\begin{abstract}
Technologies such as electrification, informatization, and intelligence will reshape the energy form of cities in the future. Energy will become a key link in sustainable development and be integrated into sustainable urban development. Starting from the key elements of the energy system for sustainable urban development, considering urban resource endowment and energy demand, combining future urban forms and technological development, from the perspective of P2C (production to consumption) \& C2P (consumption to production), a smart energy network ecosystem created by global partners and stakeholders is proposed. The features of the proposed ecosystem are: (1) the wisdom of various participants, the consideration of the renewable energy resources available in cities and towns, and the waste heat and wastewater available in urban are integrated; (2) the integrated integration of smart grid and energy storage applications; considering the integration of big data, artificial intelligence and other technical means are considered; (3) focusing on the energy supply and consumption needs of the prosumers, we will jointly customize the smart energy network ecosystem, realize the complementary use of multiple clean energy sources and the coordinated response of the source, network, and storage to provide fair and diversified comprehensive energy services for urban users. This paper demonstrates the ecosystem's modelling ideas, principles, methods and logical framework. Based on the case of the Smart Otaniemi project in Espoo, Finland, it analyses the implementation plan of the smart energy network ecosystem to provide support for the sustainable development of towns.
\end{abstract}

\section{INTRODUCTION}

Coping with megatrends such as climate change and accelerating urbanization, and the development of new technologies such as electrification, informatization, and intelligence, which will reshape the energy form of cities in the future. The large-scale, high-proportion of renewable clean and clean energy with significant randomness, intermittent news, and volatility are connected to the power grid at the energy supply side, which brings new challenges to the energy system's balanced regulation and safe and stable operation. At the energy demand side, the access of a large number of distributed power sources, micro-grids, electric vehicles, and new interactive energy-using equipment has turned energy consumers into producers and consumers and changed the traditional one-way flow pattern of source-grid-charge-user.

*Research supported by Ministry of Science and Technology National Key R\&D Program Intergovernmental International Science and Technology Innovation Cooperation Key Project (2018YFE96500) and the foundation of the Green\&low-carbon Team of Research Center for Economy of Upper Reaches of the Yangtze River in Chongqing Technology and Business University (CJSYTD702).

Chunyan Dai, Min Tang and Ying Yang are with the Research Center for Economy of Upper Reaches of the Yangtze River and the Research Centre for Enterprise Management, Chongqing Technology and Business
The complexity and uncertainty of energy system operations have increased significantly. In addition to these challenges, new opportunities are also emerging. The development of smart meters, energy routers, Internet of Things (IoT), edge computing, blockchain, artificial intelligence and other supporting equipment and emerging technologies has provided energy production and consumption in new flexibility. How to meet these emerging changes, efficiently design the smart energy network ecosystem, and integrate it into the sustainable development of the key links into the sustainable development of the city, to provide urban users with fair and diversified comprehensive energy services is a question we need to think about.

The study will introduce the key elements of the energy including for sustainable urban development, the consideration urban resource endowment and energy demand, the combination of the future urban forms and technological development and propose global cooperation from a two-way perspective of P2C \& C2P. The smart urban energy network ecosystem created by partners and stakeholders and based on the case of the Smart Otaniemi project in Espoo, Finland, analyses the implementation plan of the smart energy interconnected system to provide a reference for the sustainable development of towns.

The rest of the paper is organized as follows: The existing researches in the related work such as integrated energy services, smart energy network etc. are reviewed in Section 2. The principle and framework of the smart energy network ecosystem's designing are proposed in Section. The overall methodology and key technologies were reported in Section 4. Section 5 introduces a case study of the Smart Otaniemi of Espoo for this ecosystem's solution. Section 6 concludes the paper.

\section{RELATED WORK}

\section{A. Overview of the Smart City and Integrated Energy} Services

With the rapid development of digitalization, the relationship between people and the city has transformed from passive to positive communications, eco-sustainable vision related to the city transformation approach has been introduced by many researchers to promote a respectful urban and

University, Chongqing 400067, China. (corresponding author e-mail:( amint@163.com).

Ying Liu is with the Institute of Mechanical and Manufacturing Engineering, School of Engineering, Cardiff University, Cardiff, UK

Jijiang He is with the School of Social Sciences, Tsinghua

University,Beijing 210000,China.

Zhongqing Yang is with the Department of mechanical engineering, Aalto University, Espoo Fi-00076, Finland. 
industrial development [1, 2]. The state-of-the-art definition of a smart city has been elaborated to six correlated sections, including the smart economy, smart people, smart governance, smart mobility, smart environment, and smart living [3].

The supply structure is one of the most vital elements that influence the energy systems and environment. Through the continuous development of the Energy Internet, a convergence of distributed energy sources, diverse forms of energy including gas, heating, cooling, and electricity, and supported by the data internet, will lead to a sustainable multi-energy system [4]. He et al. (2019) [5] studies ways to improve the capacity of renewable energy to reduce the adverse effects of renewable energy generation on the power grid system, improve energy efficiency, and implement comprehensive complementary utilization of multiple energy sources. The key technologies of multi-energy complementary were discussed, including various power characteristics, complementary ways and future research directions.

The integration of different technologies and different IT systems needed to build smart city applications and services remains the most challenge to overcome. Pieroni et al. (2018) [6] proposed solution consists of using the Blockchain technology to join the grid, exchanging information, and buy/sell energy between the involved nodes (energy providers and private citizens), using the blockchain granting ledger.

In recent years, the Energy Internet technology advancement has greatly accelerated the integration of terminal energy systems, further promoting the development of multi-energy services. Meanwhile, the national new urbanization strategy in China also creates broad market space for the supply of multi-energy services. Based on the analysis on building energy consumption, F. Tang et al. (2019) [7] conduct cost-effectiveness analysis on electric heating, natural gas cooling, heating and electricity triple supply, biomass cogeneration. Then this research forecasts the market potential of multi-energy services. It is estimated that the market potential of multi-services for buildings in China from 2019 to 2021 will be approximately 408.7 billion RMB yuan.

\section{B. Smart Energy Network and Problems Solving: Challenges and Enabling}

Based on traditional energy system (TES), the Energy Internet (EI) has been discussed and extended by many researchers globally for years and reviewed by Cao et al. (2018) [4]. EI is recognised as a new energy ecosystem with better interconnectedness, openness, and flexibility, aiming to accommodate deep-penetration, clean energies; improve energy efficiency, and create a novel sharing economy to significantly reduce the cost of energy consumption.

$\mathrm{Li}$ et al. (2018) [8] summarised the integrated model of the Integrated energy distribution system (IEDs), which is regarded as a significant strategic practice on China's energy system development. Through explored the energy operation characteristics and the integrated model of IEDS, the author explained some essential issues including joint planning, optimisation control, and security analysis, state estimation and situational awareness and generalised demand-side management for the integrated energy system.

Based on the Energy Management System (EMS) for Electric power systems (EPSs), Guo et al. (2018) [9] proposed the Integrated Energy Management System (IEMS) as the brain of the EI to manage the multi-energy flow such as electricity, heating, cooling, gas, and transportation. To solve the increasing complexities of energy internet integrated with distributed renewable energy resources, Si et al. (2018) [10] deployed a more effective multi-energy management method based on the complementarity of multi-energy demand to explore optimal energy scheduling problems of prosumers. The simulation results of an urban multi-energy system exhibited that the proposed multi-energy management method could efficiently resolve the optimal energy scheduling problems to establish a win-win relationship for prosumers and generate substantial benefits for the whole system. Furthermore, Machado et al. (2018) [11] introduced a set of data-driven models, embedded in the home energy management system (HEMS) for improving renewable energy forecasting skills and modelling multiperiod flexibility of distributed energy resources for prosumers in a smart grid ecosystem.

Due to a distributed structure of present and future power systems, the Internet of Things (IoT) environment is needed to fully explore flexibility potential from the endusers and prosumers, to offer a bid to involved actors of the smart energy system. Babar et al. (2018) [12] applied a new approach to connecting the market-driven (bottom-up) DR program with the current demand-driven (top-down) energy management system. Three different functional blocks have been composed and performed as an IoT platform logical interface according to the LonWorks technology.

\section{SUPPORTING SMART ENERGY NETWORK ECOSYSTEM DESIGN -PRINCIPLE AND FRAMEWORK}

\section{A. Principle}

An important function of the smart energy network is to effectively support the sustainable development of urban. It is necessary to fully identify urban's resource endowments, real dilemmas, and future scenarios of cities and towns. Focusing on customers, the smart city's hot and cold energy providers and energy transmission companies, Communication companies, building owners, technology supporters, etc. dock on the platform. To provide diversified energy products and services, and serve the sustainable development of smart towns adapting to local conditions. Therefore, the design idea of the smart energy network ecosystem is:

(1) This is an open and innovative energy network ecosystem that is fully integrated with the sustainable development of the city This ecosystem integrates the idea of collaborative innovation on the Internet, share data, share innovation, link global partners and stakeholders, energy 
system construction and operation agencies at all levels, industry-university-research institutes, third-party service providers, and application development agencies, etc. The new standards, new technologies and new models for smart energy network ecosystems, to realise collaborative innovation and sustainable development of energy interconnected systems, and better achieve the complementary use of multiple clean energy sources and the coordinated response of source, grid, and storage are jointly customised. Furthermore, fair and diversified comprehensive energy services to urban users are provided.

(2) The local conditions, maximise the use of clean energy inside and outside of urban and achieve sustainable energy supply need to be adapted. Based on the concepts of efficient and clean energy substitution and re-electrification, the end demands of various energy categories, and consider the urban energy resources and current and future demand conditions, and consider the urban energy resource conditions (such as wind power, photovoltaic power, solar energy, ground-source heat pumps, solid waste, biomass, municipal wastewater, urban waste heat, peak valley power) and external resources that can be obtained through channels such as large power grids, and optimise the balance between internal and external resources to maximise the use of clean energy are analysed.

(3) The users and fully meet users' energy supply and energy consumption requirement needs to be focused on. In particular, energy prosumers. who may consume energy and produce energy themselves. have entered the market. They have changed the one-way flow model of the traditional energy trend from source-network-charge-users. It is necessary to fully stimulate new business formats, new models, and new applications to form an urban energy commercial ecosystem. When designing a smart energy network ecosystem, marketoriented, customer-centric, and extensive participation of participants, fully consider the integration of big data, blockchain, artificial intelligence and other technical means carry out business model innovation, and form a cover of energy production, transmission, trading, and utilisation of the full chain, including urban energy commercial ecosystems in key formats such as system optimisation and operation and maintenance, comprehensive solution provision, data management, application development, and financial services need to be considered.

(4) The deep integration of the energy system with the outside, and fully consider the integrated planning and design need to be studied, which includes: The first part is Physical information integration: Physical information fusion is the basis of the intelligentization of smart urban energy systems. The fusion standard of energy devices and information collection equipment, and the collaborative design of energy networks and information networks are the keys to physical information integration. The second part is the energy and transportation integration of the energy system, and the transportation system is the key subsystems of urban. The matching of the layout of charging piles, the control of the charge and discharge of electric vehicles, and the control of traffic flow is the core of energy and transportation integration planning. The third part is the integration of energy supply and demand. Distributed renewable energy power generation/heating, small-scale combined heating and cooling, small geothermal, urban wastewater, urban waste heat, P2G, V2G, heat storage / cold storage/gas storage/power storage, smart home, smart buildings, charging the various elements such as piles are organically combined to build a smart energy network to realise on-demand conversion and coordination and mutual benefit among multiple energy forms such as electricity, gas, heat, and cold in the network.

(5) The ways of participating in large-scale systems dispatch operations such as distributed generation, micro-grid, electric vehicles, energy storage, new interactive energy-using equipment, and demand response, and take into account the participating systems' power balance, peak and frequency regulation, and backup planning and design of the integrated smart energy interconnection system in cities and towns under the conditions of potential and promotion of clean energy consumption are fully considered.

Therefore, the modelling principles for smart energy interconnected systems are as follows:

- 1) The application context is close to the terminal energy network level on the user side. The technological, economic and social development, verify existing stock and reasonably determine future increments are fully considered.

- $\quad$ 2) The application context is not restricted by regions. Relying on the Internet and modern ICT technology to achieve full network coverage and support phased and cross-region construction.

- 3) Multi-energy complementary comprehensive integration management needs to be achieved. As the bottom layer and application technology support of integrated energy services, it serves multiple energy complementarities and supports multiple energy forms such as smart grid, wind, light, storage, waste heat, and wastewater.

- 4) The architecture has flexible and expandable capabilities, which can be divided into energy supply foundation, communication network, data collection, cloud computing platform, cloud functions, business applications. The architecture is designed according to commonality and modularity, with good distribution implementation capabilities and flexible expansion capabilities on demand, that is, the system architecture has good flexibility.

- 5) The communication network has a flexible and reliable Internet of Things accesses capabilities.

- 6) Use the cloud platform to build a smart energy integrated service system platform. Build a private + public, hybrid cloud structure based on business characteristics and scenario requirements. The system architecture has flexible and expandable capabilities; the cloud platform has user-side access and service capabilities and provides customised services and resource rental services on demand for different types of users. Functions such as energy regulation, grid operation and maintenance, asset management, and 
marketing services are moved to the cloud in the form of PaaS and SaaS, respectively.

- $\quad$ 7) Blockchain, big data, edge computing and other technologies are fully applied. Based on cloud computing as the computing power support, the technology is empowered with technologies such as big data, artificial intelligence, the Internet of Things, $5 \mathrm{G}$ communications, and the value reconstruction and transfer of energy blockchain technology play the core role.

\section{B. Framework}

The smart energy network ecosystem based on "multienergy integration", which is the endowment of local energy resources, integrated the wisdom of experts in various fields. It relies on the energy intelligence perception and trading platform to build a smart energy system to achieve the purpose of sustainable urban energy supply. It has "Four-tier architecture" which are:1) Multi-energy conversion and complementary energy production and physical consumption layer, 2.) Smart energy data sensing and transmission layer, 3) Multi-energy production and consumption two-way transaction system layer, 4) Multi-energy production and consumption complex scene application layer. The technology evolution route based on "real-world vision" and the core architecture based on "computing power value". The main idea of business logic is value-added. Figure 1 shows the framework of the smart energy network ecosystem.

\section{Methodology}

\section{A. Multi-energy complementary integration \& Source- network-load-storage collaboration}

The multi-energy complementary system should have the following characteristics:

The combination of multiple energy systems in the horizontal direction and the coordinated optimisation of distributed "source-grid-charge-storage" in the vertical direction. Under the high renewable energy penetration rate, considering the random characteristics of real-time electricity prices, changes in operating modes, demand response, and open markets, the uncertainty of the system is further increased, and coupled with the complementary coupling of the system, the scale of the energy system is doubled. It has wider openness and greater system complexity.

The application of virtual cloud technology integrates the existing energy dispatch management platform, enterprise energy monitoring and evaluation platform, demand-side management platform, and user energy optimisation terminal, and merges into a multi-dimensional and multi-energy flow physical information system for distributed power, CCHP, a variety of energy storage, electric vehicles, etc. It also provides diversified physical interfaces, which can accurately measure, summarise, store, and analyse predicted energy consumption data, energy price information, and the overall operating situation of multi-energy system operations, forming operational safety and economical energy Interactive and orderly integrated energy service platform.
Multi-energy unified dispatching is achieved through a multi-energy unified energy system regulation sub-platform.

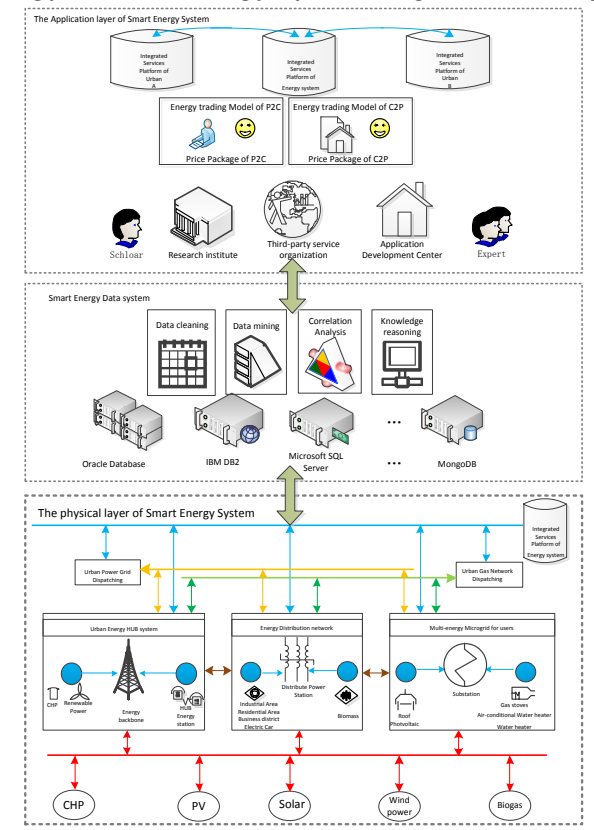

Figure 1. the framework of the smart energy network ecosystem

Through the establishment of network volatility and stochastic smoothing strategies and risk control strategies, the cluster load response characteristics and the conversion between multi-energy, and exerting the role of energy storage in balancing energy space-time differences for multi-energy unified regulation is fully considered. At the same time, to ensure the safe and uniform dispatch of the entire city's energy system, the dispatch priority of the energy system regulation sub-platform is higher than the dispatch of each energy type of the energy distribution network.

\section{B. Key technologies}

The main energy system related a lot of challenges, such as transitioning from centralised energy system towards distributed energy system for improved reliability, resilience and cost efficiency; Enabling and motivating passive consumers to become active prosumers; Increasing share of renewable energy production by allowing demand-side flexibility. The following technologies are adopted:

- AI-based prediction of energy production and consumption on different levels of the energy system.

- DLT/Blockchain which enabling dynamic and realtime energy markets and flexibility control trade transactions.

- $\quad$ AI-based optimal and efficient energy and flexibility management.

- $\quad$ AI \& DLT/Blockchain toolkit for the smart energy network stakeholders. In the smart energy network ecosystem, which requires new kinds of stakeholder interaction, interworking socio-cyber-physical systems architecture with new AI \& DLT based 
enablers for prediction and control. The intermediary results include: Deep Neural Networks (DNN) based 24h ahead forecasting model of district heating and electricity demand based on weather forecast; Local PV solar power generation modelling \& Forecasting ongoing using hierarchical double-stage AI-based approaches.

\section{The business logic of value-added services}

\section{1) Open Sharing Platform}

A data open sharing subsystem that fully stimulates multiparty participation needs to be created, which is conducive to

\section{3) Application Storage Platform}

Aiming at the long-term goal of forming a smart energy application App operation platform in urban, an application storage platform that meets users' diverse service needs need to be set up. An application warehouse according to the business chain of "application access-review release-user loading" to form a smart energy application ecosystem in cities and towns to ensure the safe and efficient operation of smart energy systems in cities also need to be built.

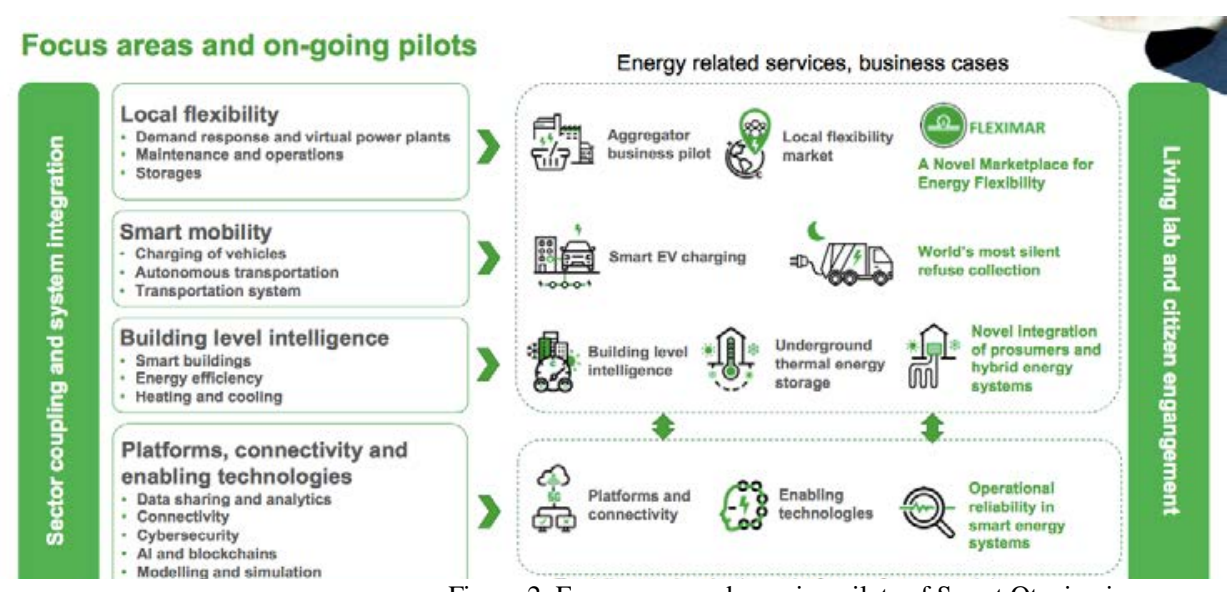

Figure 2. Focus areas and ongoing pilots of Smart Otaniemi

spawning new formats and models. Firstly, the government level encourages the open sharing of multiple parties' data through the formulation of policy mechanisms, including energy consumption data, transaction data, and system operation data. Secondly, build corresponding data security policies, verification strategies, and risk prevention mechanisms, and improve data security and data quality through data cleaning and logical verification. Finally, it is widely shared to guide and stimulate the enthusiasm of all parties to participate in the construction of urban smart energy systems and promote new business formats. The data is shared with energy system construction and operation agencies, industry-university-research institutes, third-party service providers, application development agencies, etc. at all levels, guide all parties to participate, give play to the initiative of all parties, and help promote new models and new ideas.

\section{2) Energy Trading Sub-platform}

A user-centric energy trading sub-platform with new business formats such as electric vehicles as key elements of the market needs to be created. The energy trading subplatform mainly provides diversified energy trading methods, which can meet the diversified energy trading needs of users and enterprises, such as B2C interactive transactions and price packages, B2B bilateral contracts, centralised matching, standard contracts, electronic auctions, etc. The energy transaction agreement comes into effect in conjunction with the generation of regulatory instructions to meet the efficient transactions of multiple entities and multiple energy varieties.

\section{SMART ENERGY NETWORK ECOSYSTEM'S DESIGN AND OPERATION: A CASE STUDY}

In the Smart Otaniemi (Smart Otaniemi Project) showroom in Espoo's Finnish National Technology Research Centre (VTT) Co., Ltd., you can see pilot projects including smart energy and ICT projects. The Finnish Ministry of Commerce has allocated nearly five million Euros for the development of a smart and flexible energy system test platform at Smart Otaniemi in Espoo. The project involved 11 companies and three research institutes. Besides, more than 20 companies will use their funds to participate in the development of the test platform. Smart Otaniemi is an innovation ecosystem that connects experts, organisations, technologies, and pilots. It provides an innovative ecological cooperation framework for the top players in the smart energy field to develop and collaborate on innovation. Furthermore, it allows Finland and global partners to build a new Smart energy system. The first experiment of the system began in 2018, mainly to gather information and experience on the use of $5 \mathrm{G}$ and IoT platforms to improve the energy efficiency of buildings, more flexible electricity markets and the possibility of charging electric vehicles. Otaniemi's smart energy test platform is part of the "Finnish Enterprise" smart energy program, which uses test platforms created in different regions of Finland to accelerate Finnish companies' know-how and competitiveness in the international market. The development of new, user-friendly energy services by actively engaging consumers in testing and developing solutions are shown in Figure 2.

The services enable Espoo to work with residents, businesses and the research community to formulate service 
plans and use artificial intelligence and data analysis to promote digital equality and social inclusion. For example, the Omnia AI Lab, an adult education program in Espoo, trained the world's first 100 senior citizen AI mentors to promote digital equality. Students from the Aalto University campus will also participate in the test solution. Students can also participate in development work through possible thesis topics. It was very successful to use Espoo's energy system's planning, which is shown in Figure 3.

\section{CONCLUSION}

The sustainable development of urban needs to fully consider the development of future technologies and the future shape of cities, the endowment of urban resources and access to sustainable energy, and the needs of users to realise commercial value-added. This paper starts from the key elements of the sustainable development of the urban energy system, combines the future urban form and technology development from the perspective of P2C \& C2P, a global partner and stakeholders to create a sustainable energy ecosystem was proposed. The system integrates the wisdom of multiple participants, focusing on the energy supply and user needs of producers and consumers. Furthermore, it jointly customises a smart energy interconnection system to realise the complementary use of multiple clean energy sources and the coordinated response of the source network to provide fair

and diverse urban users comprehensive energy services. This idea has been applied prototype of the Smart Otaniemi project case in Espoo, Finland. The construction concept and method of this energy interconnected ecosystem can provide support for the sustainable development of urban.

Figure 3. Espoo's energy designing and operation based on Smart Otaniem

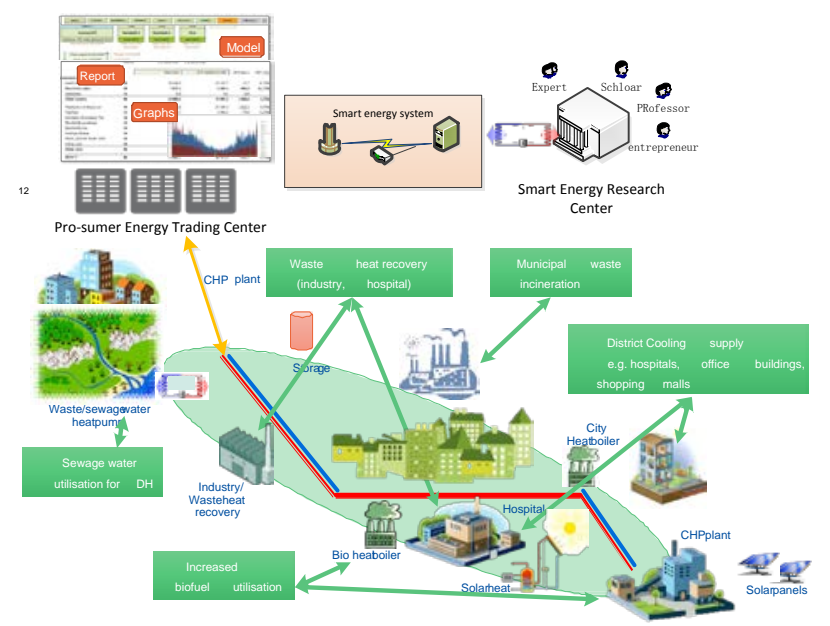

\section{ACKNOWLEDGEMENT}

The authors would like to thank for the partnerships: Dr.Johan Wallin in Synocus Oy and Mr Penttila Kalle-Erkk in Fortum Oy, Who provide the information and the figures about the project in Espoo, Finland. And also thank for the editor and the anonymous reviewers for their valuable comments and suggestions. Financial support from the Ministry of Science and Technology National Key R\&D Program Intergovernmental International Science and Technology Innovation Cooperation Key Project "EIR Program - New Urban Energy Interconnect System Research and it is Pilot Application (Project No.: 2018YFE96500)" and the Green\&low-carbon Team of Research Center for Economy of Upper Reaches of the Yangtze River in Chongqing Technology and Business University (CJSYTD702)

\section{REFERENCES}

[1] E. O'Dwyer, I. Pan, S. Acha, and N. Shah, "Smart energy systems for sustainable smart cities: Current developments, trends and future directions," Applied energy, vol. 237, pp. 581-597, 2019.

[2] M. Mohammadi, Y. Noorollahi, B. Mohammadi-ivatloo, M. Hosseinzadeh, H. Yousefi, and S. T. Khorasani, "Optimal management of energy hubs and smart energy hubs-a review," Renewable and Sustainable Energy Reviews, vol. 89, pp. 33-50, 2018.

[3] B. N. Silva, M. Khan, and K. Han, "Towards sustainable smart cities: A review of trends, architectures, components, and open challenges in smart cities," Sustainable Cities and Society, vol. 38, pp. 697-713, 2018.

[4] Y. Cao et al., "A comprehensive review of Energy Internet: basic concept, operation and planning methods, and research prospects," Journal of Modern Power Systems and Clean Energy, vol. 6, no. 3, pp. 399-411, 2018.

[5] X. He and L. Zheng, "Analysis Of Multi-energy Complementary Integration Optimization Technology," in E3S Web of Conferences, 2019, vol. 118, p. 01057: EDP Sciences.

[6] A. Pieroni, N. Scarpato, L. Di Nunzio, F. Fallucchi, and M. Raso, "Smarter city: smart energy grid based on blockchain technology," Int. J. Adv. Sci. Eng. Inf. Technol, vol. 8, no. 1, pp. 298-306, 2018.

[7] F. Tang, H. Dai, and Y. Jian, "Multi-Energy Services for Buildings in China: Cost-Effectiveness Analysis and Market Potential Forecast," in 2019 IEEE International Conference on Energy Internet (ICEI), 2019, pp. 444-448: IEEE.

[8] D. Wang et al., "Review of key problems related to integrated energy distribution systems," CSEE Journal of Power and Energy Systems, vol. 4, no. 2, pp. 130-145, 2018.

[9] H. Sun et al., "Integrated energy management system: Concept, design, and demonstration in China," IEEE Electrification Magazine, vol. 6, no. 2, pp. 42-50, 2018.

[10] F. Si, J. Wang, Y. Han, Q. Zhao, P. Han, and Y. Li, "Cost-efficient multi-energy management with flexible complementarity strategy for energy internet," Applied Energy, vol. 231, pp. 803-815, 2018.

[11] R. J. Bessa et al., "Data Economy for Prosumers in a Smart Grid Ecosystem," in Proceedings of the Ninth International Conference on Future Energy Systems, 2018, pp. 622-630.

[12] M. Babar, J. Grela, A. Ożadowicz, P. H. Nguyen, Z. Hanzelka, and I. Kamphuis, "Energy flexometer: Transactive energy-based internet of things technology," Energies, vol. 11, no. 3, p. 568, 2018. 